\title{
IMMERSION OF MANIFOLDS OF NONPOSITIVE CURVATURE
}

\section{BARRETT O'NEILL}

In [4] Tompkins proved that a flat compact Riemannian manifold $M$ of dimension $n$ cannot be (isometrically) immersed in Euclidean $(2 n-1)$-space. Chern and Kuiper conjectured in [2] that the result holds if the Riemannian (i.e. sectional) curvature $K$ of $M$ is never positive. An algebraic result verifying this conjecture was found by Otsuki [3]. We shall prove:

THEOREM. Let $M$ be a compact $n$-dimensional Riemannian manifold and let $\bar{M}$ be a complete simply connected Riemannian manifold of dimension less than $2 n$. If the Riemannian curvatures $K$ and $\bar{K}$ of $M$ and $\bar{M}$ satisfy $K \leqq \bar{K} \leqq 0$, then $M$ cannot be immersed in $\bar{M}$.

Simple examples involving spheres and tori show that the theorem fails if either inequality or the simple connectedness of $\bar{M}$ is deleted.

Following [1] we express the second fundamental form information of an immersion $i: M \rightarrow \bar{M}$ in terms of the difference transformation $T$, a function which assigns to each vector $x$ in $M_{m}$ (the tangent space to $M$ at $m \in M)$ a linear transformation $T_{x}$ from $M_{m}$ to the orthogonal complement $M_{m}^{\perp}$ of $d i\left(M_{m}\right)$ in $\bar{M}_{i(m)}$. The natural definition of $T$ is in terms of the notion of difference of two connections [1], however it may be described in terms of the classical second fundamental form $S$ as follows: $S$ is a function which assigns to each vector $z \in M_{m}^{\perp}$ a symmetric linear operator $S_{z}$ on $M_{m}$. If $x \in M_{m}$ and $z \in M_{m}^{\perp}$, let $T_{x}(z)=S_{z}(x)$; then uniquely extend $T_{x}$ to be a skew-symmetric operator on all of $\bar{M}_{i(m)}$. For our purposes, as indicated above, we need only the portion of $T_{x}$ defined on $d i\left(M_{m}\right)$ or, equivalently, $M_{m}$. As a function of $x, y \in M_{m}, T_{x}(y)$ is bilinear and symmetric.

The difference transformation relates the Riemannian curvatures of $M$ and $\bar{M}$ as follows: if $x$ and $y$ span a plane $P$ in $M_{m}$, then

$$
K(P)=\frac{\left\langle T_{x}(x), T_{y}(y)\right\rangle-\left\|T_{x}(y)\right\|^{2}}{\|x \wedge y\|^{2}}+\bar{K}(d i(P)) .
$$

This formula, the Gauss equation, is readily obtained from the second structural equations of $M$ and $\bar{M}$.

The following lemma extends a well-known Euclidean fact.

Lemma 1. Let $i: M \rightarrow \bar{M}$ be an immersion of a compact Riemannian

Received by the editors March 20, 1959. 
manifold in a complete simply connected manifold with Riemannian curvature $\bar{K} \leqq 0$. Then there is a point $m \in M$ and a vector $z \in M_{m}^{\perp}$ such that $\left\langle T_{x}(x), z\right\rangle<0$ for all nonzero $x \in M_{m}$.

Proof. Fix a point $\bar{m}$ in $\bar{M}$ and use the following notation: $m$, a point of $M$ such that $i(m)$ has maximum distance from $\bar{m} ; \sigma:[0,1]$ $\rightarrow \bar{M}$, the unique geodesic from $m$ to $i(m) ; z$, the velocity vector $\sigma(1)$ of $\sigma$ at $i(m)$. If $x \in \operatorname{di}\left(M_{m}\right)$ there is a differentiable map $r:[0,1]$ $\times[0,1] \rightarrow M$ such that (1) $r(\cdot, 0)=\sigma$, (2) for each $v \in[0,1], r(\cdot, v)$ is a geodesic, (3) $r(0, \cdot)=\bar{m}$ and $r(1, \cdot) \in i(M),(4)$ if $X$ is the vector field on $\sigma$ such that $X(u)$ is the velocity of $r(u, \cdot)$ at $v=0$, then $X(1)=x$. Let $l(v)$ be the length of $r(\cdot, v)$, that is, the distance from $\bar{m}$ to $r(1, v) \in i(M)$. Obviously $l^{\prime}(0)=0$ and $l^{\prime \prime}(0) \leqq 0$. The vanishing of the first variation implies $z \in M_{m}^{\perp}$. By the Synge formula for the second variation [1] we have

$$
s l^{\prime \prime}(0)=\int_{0}^{1}\left\{\left\|X^{\prime}\right\|^{2}-\bar{K}(\dot{\sigma}, X)\|\dot{\sigma} \wedge X\|\right\}+\left\langle T_{x}(x), z\right\rangle
$$

where $s$ is the length of $\sigma$, and $X^{\prime}$ is the covariant derivative of $X$. Since $\bar{K} \leqq 0$, the integral term is positive if $x \neq 0$, hence $\left\langle T_{x}(x), z\right\rangle<0$.

Proof of The theorem. Following Tompkins' scheme we reduce the proof to a problem in linear algebra. Let $i: M \rightarrow \bar{M}$ be an immersion, where $M$ and $\bar{M}$ are as described in the theorem, except that no restriction is made on the dimension of $\bar{M}$. Let $m$ and $z$ be as in Lemma 1. From the formula (1) and the condition $K \leqq \bar{K}$ we get $\left\langle T_{x}(x), T_{y}(y)\right\rangle \leqq\left\|T_{x}(y)\right\|^{2}$ for all $x, y \in M_{m}$. We need only prove that dimension $M_{m}^{\perp} \geqq n$, and this follows from

Lemma 2. Let $U$ and $V$ be finite-dimensional real vector spaces, $V$ with an inner product. Suppose that for each $x \in U$ there is a linear transformation $T_{x}: U \rightarrow V$ such that:

(1) $\left\langle T_{x}(x), T_{y}(y)\right\rangle \leqq\left\|T_{x}(y)\right\|^{2}$ for all $x, y \in U$.

(2) $T_{x}(y)$ is bilinear and symmetric in $x$ and $y$.

(3) There is a vector $z \in V$ such that $\left\langle T_{x}(x), z\right\rangle<0$ for all nonzero $x$ in $U$. Then dimension $V \geqq$ dimension $U$.

Proof. Suppose the contrary; then for every $u \in U$ there is a nonzero $v \in U$ such that $T_{u}(v)=0$, hence $\left\langle T_{u}(u), T_{v}(v)\right\rangle \leqq 0$. Consider the real-valued function $f$ on $U-\{0\}$ for which

$$
f(u)=\frac{\left\langle T_{u}(u), z\right\rangle}{\left\|T_{u}(u)\right\|\|z\|} .
$$

Since $f$ is continuous and constant on lines through the origin, it has a 
minimum, say $f(x)$. Let $y$ be a nonzero vector such that $T_{x}(y)=0$ and $\left\langle T_{x}(x), T_{y}(y)\right\rangle \leqq 0$. Note that $T_{x}(x)$ and $T_{y}(y)$ are independent, for otherwise we may assume $T_{x}(x)+T_{y}(y)=0$, which contradicts (3). Let $P$ be the plane spanned by these two vectors, and let $z^{\perp}$ be the set of vectors orthogonal to $z$. Since $T_{x}(x)$ is not in $z^{\perp}$, the subspace $P \cap z^{\perp}$ is 1-dimensional. Let $p$ be the unique unit vector in $P$ such that $\langle p, z\rangle\left\langle 0\right.$ and $p$ is orthogonal to $P \cap z^{\perp}$. Clearly $\langle p, z\rangle\langle\langle q, z\rangle$ if $q$ is a unit vector in $P$ different from $p$. Now the definition of $p$ together with the inequalities $\left\langle T_{x}(x), T_{y}(y)\right\rangle \leqq 0,\left\langle T_{x}(x), z\right\rangle<0,\left\langle T_{y}(y), z\right\rangle<0$ imply that $p$ lies between $T_{x}(x)$ and $T_{y}(y)$, that is, that we may write $p=\lambda^{2} T_{x}(x)+\mu^{2} T_{y}(y)$. Thus $p=T_{\lambda x+\mu y}(\lambda x+\mu y)$. In view of the minimality properties of $p$ and $T_{x}(x)$ we must have $T_{x}(x)$ a positive scalar multiple of $p$. But since $p$ is orthogonal to $P \cap z^{\perp}$, this implies $\left\langle T_{y}(y), z\right\rangle$ $\geqq 0$, a contradiction.

It is clear that the theorem holds in slightly more general form, patterned on the full Chern-Kuiper conjecture, namely: if there is at each point $m$ of $M$ a $q$-dimensional subspace $S_{m}(q \geqq 2)$ of $M_{m}$ such that $K \leqq \bar{K}$ holds when $K$ is restricted to planes in any $S_{m}$, then immersion is impossible if the dimension of $\bar{M}$ is less than $n+q$.

\section{REFERENCES}

1. W. Ambrose, The use of the structural equations in the classical calculus of variations (to appear in the J. Indian Math. Soc.)

2. S. S. Chern and N. H. Kuiper, Some theorems on the isometric imbedding of compact Riemann manifolds in Euclidean space, Ann. of Math. vol. 56 (1952) pp. 422-430.

3. T. Otsuki, On the existence of solutions of a system of quadratic equations and its geometrical application, Proc. Japan. Acad. vol. 29 (1953) pp. 99-100.

4. C. Tompkins, Isometric embedding of flat manifolds in Euclidean space, Duke Math. J. vol. 5 (1939) pp. 58-61.

Massachusetts Institute of Technology 